\title{
Article \\ Maternal Mental Health under COVID-19 Pandemic in Thailand
}

\author{
Wachiranun Sirikul, Krongporn Ongprasert $(D)$, Chanodom Piankusol (D) and Penprapa Siviroj * (D)
}

check for

updates

Citation: Sirikul, W.; Ongprasert, K.; Piankusol, C.; Siviroj, P. Maternal

Mental Health under COVID-19 Pandemic in Thailand. Int. J. Environ. Res. Public Health 2022, 19, 347. https://doi.org/10.3390/ ijerph19010347

Academic Editors: Javier Alvarez-Galvez, Diego Gomez-Baya, Carolina Lagares-Franco and Lawrence Palinkas

Received: 18 November 2021 Accepted: 28 December 2021 Published: 29 December 2021

Publisher's Note: MDPI stays neutral with regard to jurisdictional claims in published maps and institutional affiliations.

Copyright: (c) 2021 by the authors. Licensee MDPI, Basel, Switzerland. This article is an open access article distributed under the terms and conditions of the Creative Commons Attribution (CC BY) license (https:// creativecommons.org/licenses/by/ $4.0 /)$.

\author{
Department of Community Medicine, Faculty of Medicine, Chiang Mai University, Chiang Mai 50200, Thailand \\ wachiranun.sir@cmu.ac.th (W.S.); krongporn.o@cmu.ac.th (K.O.); chanodom.p@cmu.ac.th (C.P.) \\ * Correspondence: penprapa.s@cmu.ac.th
}

\begin{abstract}
Numerous nations have implemented lockdown measures in response to the COVID-19 pandemic. As a consequence of the lockdown on daily living, social participation, and health service accessibility, vulnerable people, for example, new mothers, may experience an increase in mental health problems. This cross-sectional survey was conducted to investigate the impact of the COVID-19 pandemic lockdown on Thai new mothers and the variables affecting their mental health. The survey data were collected from 903 Thai mothers with infants aged 0-12 months using an online platform and a face-to-face interview questionnaire survey between 17 July and 17 October 2020, during the first nationwide COVID-19 lockdown period. For the final analysis, there were 862 participants who completed all of the questions. The full exploratory analysis was performed by multivariable linear regression to identify the variables influencing maternal mental health. Our study demonstrated that new mothers reported feeling a high extent to some extent of worry $(44.9 \%)$, increased appetite $(40.4 \%)$, becoming easily annoyed or irritable $(39.1 \%)$, and feeling down $(33.5 \%)$, whereas $82.7 \%$ felt able to cope with the first lockdown situation. Practiced relaxation techniques were associated with positive maternal mental health (adjusted $\beta=1.05,95 \%$ CI 0.57 to $1.52, p<0.001)$. The perceived impact of the COVID-19 lockdown was on the household's ability to pay for rent, to make mortgage payments (adjusted $\beta=-1.59,95 \% \mathrm{CI}-2.87$ to $-0.36, p=0.011$ ), the household's ability to pay for other essentials, such as utilities and medication (adjusted $\beta=-1.99$, $95 \% \mathrm{CI}-3.16$ to $-0.81, p=0.001$ ), household crowding after lockdown (adjusted $\beta=-3.46,95 \% \mathrm{CI}$ -4.86 to $-2.06, p<0.001$ ), and not going outside or doing outdoor activities (adjusted $\beta=-2.22$, $95 \%$ CI -3.35 to $-1.08, p<0.001$ ). These impacts were significantly associated with negative mental health. In conclusion, our results emphasize the critical need for continuous monitoring of maternal mental health and developing an effective response strategy and activity for promoting maternal mental health under the stress of repetitive lockdowns and increased economic pressures.
\end{abstract}

Keywords: maternal mental health; COVID-19; lockdown impacts; activities during lockdown; illness experiences

\section{Introduction}

Since the global COVID-19 pandemic in late 2019, many countries have implemented restrictive measures such as social distancing through lockdown regulations. Social distancing is a recommended prevention strategy for the general population. It includes keeping a physical distance between people (at least one meter) and reducing contact with contaminated surfaces while encouraging and maintaining social connections within families and communities. As disease transmission began to spread to community levels, a nationwide lockdown was implemented as a public health emergency response to break the chain of transmission through movement restrictions, stay-at-home measures, and the closure of essential services (e.g., public transportation, health, and social services) [1,2]. The first confirmed case of COVID-19 reported in Thailand was on 13 January 2020, after which it continued to spread. As of 21 October 2021, there were 1,821,579 cases of COVID-19 and 18,559 deaths $(1.01 \%)$ in Thailand [3]. The first lockdown in Thailand was imposed by the 
government with a curfew for the whole population from 10 p.m. to 4 a.m. between 3 April and 15 June 2020 [4].

The consequences of COVID-19 lockdown have resulted in extensive disruption to daily life. In the US, adverse mental health problems have been associated with the COVID19 outbreak [5]. Many people have been left feeling anxious or depressed, fearful of infection, and stressed, making the future seem uncertain [6-10]. Moreover, the impact of lockdown can include the loss of income and housing and reduced support from their family, peer group, and health personnel, all of which contribute to changes in people's daily living and their mental health [11-13]. Worries resulting from the pandemic are to be expected and common, but nevertheless might cause suffering and impairment in social and occupational dimensions. In addition to this, the restrictive measures might have psychological effects, causing an emotional disturbance, anxiety, anger, depression, insomnia, and somatic signs and symptoms $[14,15]$.

Pregnant and postpartum women are vulnerable to COVID-19 [16-18]. Many mothers increased their time for childcaring but had greater stress (42\%) and increased frustration $(50 \%)$ [19]. New mothers across the world were shown to be affected by the lockdown in multiple ways, as indicated by an increase in negative emotions and psychological symptoms, including feeling down, anxious or worried, lonely, and having depressive or anxiety symptoms $[18,20,21]$.

To the best of our knowledge, there are no published studies assessing maternal mental health during the COVID-19 pandemic and lockdown in Thailand. Since established evidence points out that the COVID-19 pandemic and lockdown measures aggravate mental health problems in new mothers and their households, this study aims to investigate the impacts of the COVID-19 pandemic and the nationwide lockdown on household capabilities, daily living activities, and the mental health of new mothers living in Thailand. We also examined the association between potential influencing factors and maternal mental health in order to suggest future preventive strategies for maternal mental health.

\section{Materials and Methods}

\subsection{Study Design and Participants}

The cross-sectional study was conducted via a nationwide online survey and a faceto-face interview survey from 17 July 2020 to 17 October 2020, after the first nationwide COVID-19 lockdown period in Thailand (from 3 April to 15 June 2020) and before the second wave of the outbreak from 21 December 2020 to 11 February 2021. We recruited postpartum women over 18 years old with infants under age 12 months at the time of the survey, regardless of nationality, living in Thailand during the COVID-19 pandemic. Using an online survey enabled us to capture mothers' experiences during the pandemic in a timely and remote manner and to reach mothers from all regions of Thailand. Additionally, faceto-face interviews were performed in Northern Thailand at secondary and tertiary public hospitals as well as private hospitals, to represent a diversity of maternal socioeconomic backgrounds. Details regarding sample size, participant recruitment, and data collection have been published elsewhere [13].

For the online survey, 732 participants gave their consent to participate in this study. Of those, 426 participants (58.2\%) answered complete questions. The face-to-face interview survey had $436(92.8 \%)$ participants out of 470 who answered complete questions. For the final analysis, the sample population consisted of 862 participants, recruited independently from the online platform $(426,49.4 \%)$ and face-to-face interviews $(436,40.6 \%)$ (Figure 1). The majority of participants lived in Chiang Mai (512, 56.6\%) and Bangkok (121, 13.3\%). Figure 2 illustrates the distribution of survey responses per province. 


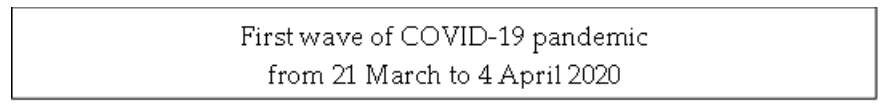

First nationwide partial lockdown from 3 April to 15 June 2020

- Restriction order (10 p.m. to 4 a.m.)

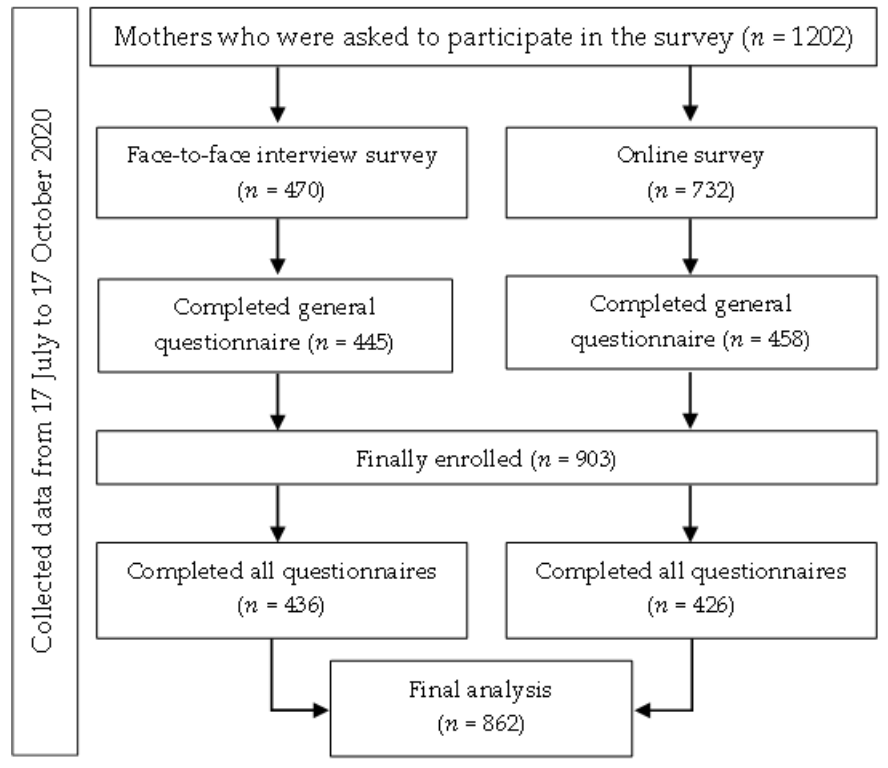

Second wave of COVID-19 pandemic from 21 December 2020 to 11 February 2021

Figure 1. COVID-19 pandemic and lockdown durations in Thailand and period of the online platforms, interviews, and sample size of this study.

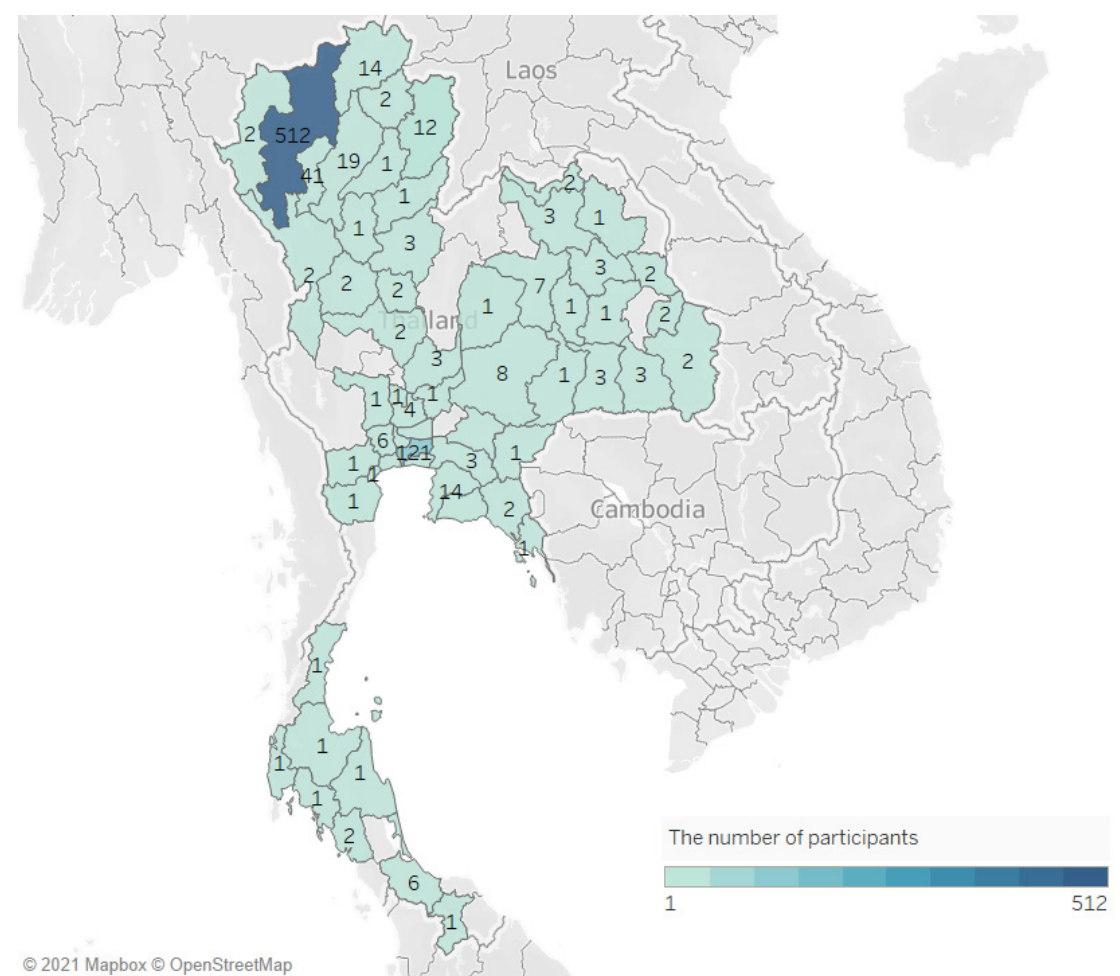

Figure 2. The distribution of survey participants. The figure was created by Tableau Desktop 2021.1, LLC, Seattle, WA, USA. 


\subsection{Questionnaire Design}

The participants were asked about their experience of COVID-19 symptoms and if they had been tested for COVID-19, the mother's activities, and moods during the COVID-19 lockdown. This study used a structured questionnaire adapted from the UK COVID-19 New Mum Survey in English [19] and translated into Thai; the pilot questionnaire was tested to improve the questions with 5 mothers at the well-baby clinic, Maharaj Nakorn Chiang Mai hospital before use. The questionnaire had the following five parts:

(1) Socio-demographic characteristics-age, ethnicity, marital status, education, employment status, household income, household members, type of accommodation (e.g., own house, apartment/condominium, dormitory, and rental house), number of rooms in the accommodation, and pets at home. Moreover, we collected the infant age, mother's alcohol drinking during the last 7 days, and smoking in the last 2 days. The answers to these questions are "yes" or "no".

(2) Experiences of COVID-19 symptoms and investigation-the questions in this section asked about the experience of COVID-19, if mothers and other members in the household had COVID-19 symptoms, and if the mothers had been investigated for COVID-19. The answers to these questions are "yes" or "no".

(3) Perception of COVID-19 lockdown impacts-the questions in this section about how the lockdown impacted mothers' life and activities in negative ways during the COVID-19 lockdown consisted of 6 questions. The answers to these questions were given on a 4-point Likert scale as major, moderate, minor, and no impact.

(3.1) "Your employment/In what ways has your work been affected by COVID-19".

(3.2) "Your partner's employment/In what ways has his work been affected by COVID-19".

(3.3) "Your household's ability to pay for other essentials, such as utilities and medication".

(3.4) "Your household's ability to pay for food".

(3.5) "Your household's ability to pay for rent/to make mortgage payments".

(3.6) "Household crowding after lockdown".

(4) Activities during the nationwide COVID-19 lockdown period-the questions in this section asked about the frequency of different activities, and the answers to these questions were given on a 4-point Likert scale as every day, more than 5 times per week, $4-5$ times per week, 1-3 times per week, and never. These questions are in two parts, as follows:

(4.1) Indoor/outdoor activities e.g., "went outside for a walk or for exercise", "went shopping at the grocery store or pharmacy", "participated in an online activity", and "practiced a relaxation technique".

(4.2) Supportive activities e.g., "contact with a mother and baby support group or breastfeeding support group", "contact with a health professional (general practioner (GP), health visitor, midwife), in person, by phone or online", and "Attended an online, phone, or in person appointment with a mental health professional".

(5) Maternal mental health-the questions in this section regarded the participants' mood during the lockdown period. This tool was a 4-point Likert scale; to a high extent, to some extent, very little, and not at all. The overall score was calculated by the sum score of positive questions (no. 1-8) minus the sum score of negative questions (no. 9-18). This individual score will represent the maternal mood status and is used as the dependent variable in the exploratory analysis by Multivariable Linear Regression. The alpha coefficient for the reliability test of the mother's mood questionnaire was 0.78 (Supplementary Table S1). There are two subsections of questions, positive and negative questions, as follows:

(5.1) Positive ways had 8 items: "I've had the opportunity to chat with my family and friends", "I feel connected with my local community", "I've enjoyed the 
weather", "I've had time to focus on my health", "I've had time to exercise", "I feel able to cope with the situation", "I feel the house chores are more equally divided among household members", and "I've had time to enjoy personal interests or hobbies".

(5.2) Negative ways had 10 items: "I've been having a poor appetite", "I've been overeating", "I've been feeling tired or having little energy", "I've been feeling worried", "I've been feeling down", "I've had trouble falling or staying asleep", "I've been feeling lonely", "I've had trouble relaxing", "I feel the house chores are less equally divided among household members", and "I've become easily annoyed or irritable".

\subsection{Statistical Analysis}

All statistical analyses were conducted using the STATA statistical software program (Stata Corp. 2019. Stata Statistical Software: Release 16, Stata Corp LLC, College Station, TX, USA). For categorical data, the maternal characteristics, perception of the lockdown impact, frequency of activities, and response to mother's mood questions were reported as the frequency with percentages. The mean with standard deviation (SD) or the $95 \%$ confidence interval $(95 \% \mathrm{CI})$ were used to present parametric data, while the median and interquartile range (IQR) were used to describe non-parametric data. The internal consistency and reliability of the maternal mental health questions were additionally evaluated by Cronbach's alpha test. The association between maternal mental health (sum scores of the maternal mental health questions) during the nationwide lockdown and influencing variables, including maternal and family characteristics, the experience of COVID-19 symptoms and investigation, the perception of the lockdown impacts, and the activity during this period, was explored using multivariable linear regression. The directed acyclic graphs were designed to illustrate the set of confounders that needed to be adjusted and to identify the factors associated with the outcome from the regression model. A regression coefficient with 95\% confidence intervals (CI) was reported to represent the magnitude of the association. The results of this study were reported according to the strengthening of the reporting of observational studies in Epidemiology (STROBE) checklist. All statistical analyses were two-sided, and a $p$-value of 0.05 was considered to be statistically significant.

\subsection{Ethical Consideration}

This study was conducted following the Declaration of Helsinki and the protocol was approved by the Research Ethics Committee, Faculty of Medicine, Chiang Mai University, Thailand (Study Code: COM-2563-07416).

\section{Results}

\subsection{Characteristics of Participants}

The characteristics of participants and their households are reported in Table 1 . The participants consisted of 903 mothers with an infant aged less than one year old. Most of the participants were in the 19-35 years age group (78.1\%) with an infant age of 6 months and younger $(63.3 \%)$. Almost all were nuclear families $(85.4 \%)$, had an employed occupation $(73.3 \%)$, and had their own house $(80.0 \%)$. About half of the mothers had education levels under a bachelor's degree (69.5\%) and 39.8\% had pets at home. A few mothers and their family members had symptoms of COVID-19 (0.9\% and $0.5 \%$, respectively), while $7.6 \%$ of the participants were tested for COVID-19. 
Table 1. The characteristics of participants and households.

\begin{tabular}{|c|c|c|}
\hline \multirow{2}{*}{ Characteristics } & \multicolumn{2}{|c|}{ Total $(n=903)$} \\
\hline & $n$ & $\%$ \\
\hline \multicolumn{3}{|l|}{ Maternal age } \\
\hline$\leq 18$ years & 2 & 0.2 \\
\hline $19-35$ years & 705 & 78.1 \\
\hline$>35$ years & 196 & 21.7 \\
\hline \multicolumn{3}{|l|}{ Infant age } \\
\hline$\leq 6$ months & 572 & 63.3 \\
\hline 6-12 months & 331 & 36.7 \\
\hline \multicolumn{3}{|l|}{ Marital status } \\
\hline Single mother & 132 & 14.6 \\
\hline Nuclear family & 771 & 85.4 \\
\hline \multicolumn{3}{|l|}{ Education } \\
\hline Below bachelor's degree & 628 & 69.5 \\
\hline Bachelor's degree or above & 275 & 30.4 \\
\hline \multicolumn{3}{|l|}{ Employment } \\
\hline Not working/Unemployed & 80 & 11.4 \\
\hline Employed & 514 & 73.3 \\
\hline Business owner & 107 & 15.3 \\
\hline Household members, mean \pm SD & 3 & \pm 1 \\
\hline \multicolumn{3}{|l|}{ Type of accommodation } \\
\hline Own house & 722 & 80.0 \\
\hline Apartment/Condominium & 46 & 5.1 \\
\hline Dormitory & 114 & 12.6 \\
\hline Rental house & 21 & 2.3 \\
\hline Number of rooms in the accommodation, mean \pm SD & 6 & \pm 2 \\
\hline \multicolumn{3}{|l|}{ Living conditions } \\
\hline Can access to a private space/garden for doing an activity & 423 & 47.8 \\
\hline Can access to a community space/garden for doing an activity & 144 & 16.0 \\
\hline Not going outside/Doing outdoor activities & 327 & 36.2 \\
\hline \multicolumn{3}{|l|}{ Household income per year } \\
\hline Less than USD 16,130 & 314 & 34.9 \\
\hline More than USD 16,130 & 588 & 65.1 \\
\hline Had pets at home & 359 & 39.8 \\
\hline Alcohol drunk during the last 7 days & 18 & 2.0 \\
\hline Smoking in the last 2 days & 2 & 0.2 \\
\hline \multicolumn{3}{|l|}{ Experience of COVID-19 symptoms and investigation } \\
\hline Had symptoms & 8 & 0.9 \\
\hline Any other member of household had symptoms & 5 & 0.5 \\
\hline Had been tested for COVID-19 & 69 & 7.6 \\
\hline
\end{tabular}

$\mathrm{SD}=$ Standard deviation.

\subsection{Mothers' Perception of Impacts of COVID-19 Lockdown}

Table 2 shows the mothers' perceptions about the impact of the nationwide COVID-19 lockdown. Mothers described their perception of the effects of COVID-19 as moderate to major most frequently for "your employment/in what ways has your work been affected by COVID-19" (47.1\%), "your household's ability to pay for other essentials, such as utilities and medication" (45.0\%), "your partner's employment/in what ways has his work been affected by COVID-19" (43.7\%), and "your household's ability to pay for food" $(42.6 \%)$ and less frequently for "your household's ability to pay for rent/to make mortgage payments" (28.9\%) and "household crowding after lockdown" (14.7\%). 
Table 2. The perception of the nationwide COVID-19 lockdown impacts.

\begin{tabular}{|c|c|c|c|c|}
\hline \multirow{3}{*}{$\begin{array}{l}\text { COVID-19 Lockdown Impacts } \\
\qquad(n=903)\end{array}$} & \multicolumn{4}{|c|}{ Levels of Impact } \\
\hline & \multicolumn{2}{|c|}{ Moderate to Major } & \multicolumn{2}{|c|}{ No or Minor } \\
\hline & $n$ & $\%$ & $n$ & $\%$ \\
\hline Your employment/In what ways has your work been affected by COVID-19 & 425 & 47.1 & 478 & 52.9 \\
\hline Your household's ability to pay for other essentials, such as utilities and medication & 406 & 45.0 & 497 & 55.0 \\
\hline Your partner's employment/In what ways has their work been affected by COVID-19 & 395 & 43.7 & 508 & 56.3 \\
\hline Your household's ability to pay for food & 385 & 42.6 & 518 & 57.4 \\
\hline Your household's ability to pay for rent/To make mortgage payments & 261 & 28.9 & 642 & 71.1 \\
\hline Household crowding after lockdown & 133 & 14.7 & 770 & 85.3 \\
\hline
\end{tabular}

\subsection{Mothers' Activities during the COVID-19 Lockdown Period}

As indicated in Table 1, most mothers had gone outdoors or had outdoor activities $(63.8 \%)$, which included going outside to a public space (15.9\%) and going to an outdoor space within the residence area $(47.8 \%)$. Table 3 shows the proportion of mothers' activities during the COVID-19 lockdown period. Considering the mothers' activities, "participated in an online activity" (41.3\%), "went outside for a walk or for exercise" (30.7\%), and "practiced a relaxation technique" $(28.8 \%)$ were most frequently rated as more than 5 times or everyday/4-5 times per week. The most frequent rating of "never to do" regarding health supportive activity from others such as health professionals and support groups were "appointment with a mental health professional" $(96.9 \%)$, "contact with a health professional (GP, health visitor, midwife), in person, by phone or online" (77.6\%), and "contact with a mother and baby support group or breastfeeding support group" $(60.9 \%)$, respectively.

Table 3. Mothers' activities during the nationwide COVID-19 lockdown period.

\begin{tabular}{|c|c|c|c|c|}
\hline \multirow{2}{*}{$\begin{array}{c}\text { Activities } \\
(n=903)\end{array}$} & \multicolumn{4}{|c|}{ Levels of Activity (Times per Week), $n$ (\%) } \\
\hline & >5 Times/Every Day & 4-5 Times & 1-3 Times & Never \\
\hline \multicolumn{5}{|l|}{ Indoor/outdoor activity } \\
\hline Participated in an online activity & $\begin{array}{c}283 \\
(31.3)\end{array}$ & $\begin{array}{c}90 \\
(10.0)\end{array}$ & $\begin{array}{c}182 \\
(20.2)\end{array}$ & $\begin{array}{c}348 \\
(38.5)\end{array}$ \\
\hline Went outside for a walk or for exercise & $\begin{array}{c}172 \\
(19.1)\end{array}$ & $\begin{array}{c}105 \\
(11.6)\end{array}$ & $\begin{array}{c}309 \\
(34.3)\end{array}$ & $\begin{array}{c}316 \\
(35.0)\end{array}$ \\
\hline Practiced a relaxation technique & $\begin{array}{c}160 \\
(17.8)\end{array}$ & $\begin{array}{c}99 \\
(11.0)\end{array}$ & $\begin{array}{c}282 \\
(31.3)\end{array}$ & $\begin{array}{c}359 \\
(39.9)\end{array}$ \\
\hline Went shopping to the grocery store or pharmacy & $\begin{array}{c}67 \\
(7.4) \\
\end{array}$ & $\begin{array}{c}101 \\
(11.2)\end{array}$ & $\begin{array}{c}501 \\
(55.5)\end{array}$ & $\begin{array}{c}233 \\
(25.8)\end{array}$ \\
\hline \multicolumn{5}{|l|}{ Supportive activity } \\
\hline $\begin{array}{l}\text { Contact with a mother and baby support group or breastfeeding } \\
\text { support group }\end{array}$ & $\begin{array}{c}83 \\
(9.2)\end{array}$ & $\begin{array}{l}58 \\
(6.4)\end{array}$ & $\begin{array}{c}211 \\
(23.4)\end{array}$ & $\begin{array}{c}548 \\
(60.9)\end{array}$ \\
\hline $\begin{array}{l}\text { Contact with a health professional (GP, Health Visitor, Midwife), } \\
\text { in person, by phone or online }\end{array}$ & $\begin{array}{c}4 \\
(0.4)\end{array}$ & $\begin{array}{c}17 \\
(1.9)\end{array}$ & $\begin{array}{c}180 \\
(20.0)\end{array}$ & $\begin{array}{c}698 \\
(77.6)\end{array}$ \\
\hline $\begin{array}{l}\text { Attended an online, phone, or in person appointment with a } \\
\text { mental health professional }\end{array}$ & $\begin{array}{c}0 \\
(0.0)\end{array}$ & $\begin{array}{c}6 \\
(0.7)\end{array}$ & $\begin{array}{c}22 \\
(2.4)\end{array}$ & $\begin{array}{c}871 \\
(96.9)\end{array}$ \\
\hline
\end{tabular}

GP $=$ General practitioner.

\subsection{The Maternal Mental Health during the COVID-19 Lockdown Period}

In Figure 3, the forest plot summarizes the participants' responses to the maternal mental health questions during the nationwide COVID-19 lockdown period. The first section of questions $1-8$ indicated a positive mood of the mother and questions 10-18 
reflected mental health from a negative perspective. The majority of mothers had a high to some extent of feelings about the opportunity to chat with family and friends $(92.0 \%)$, being able to cope with the situation $(82.7 \%)$, and appreciating the weather $(74.0 \%)$ contributed to their positive mood. In contrast, more than half of the participants responded not at all or very little about the equal distribution of house chores $(63.8 \%)$ and having time to enjoy personal interests or hobbies (63.6\%). Nevertheless, more than half of the individuals reported none or very few negative physical symptoms or moods during the lockdown. The most frequent ratings regarding a high extent or some extent of negative moods were worrying (44.9\%), increasing appetite $(40.4 \%)$, becoming easily annoyed or irritable $(39.1 \%)$, and feeling down $(33.5 \%)$. Details of the mother's mood responses are presented in Table S2.

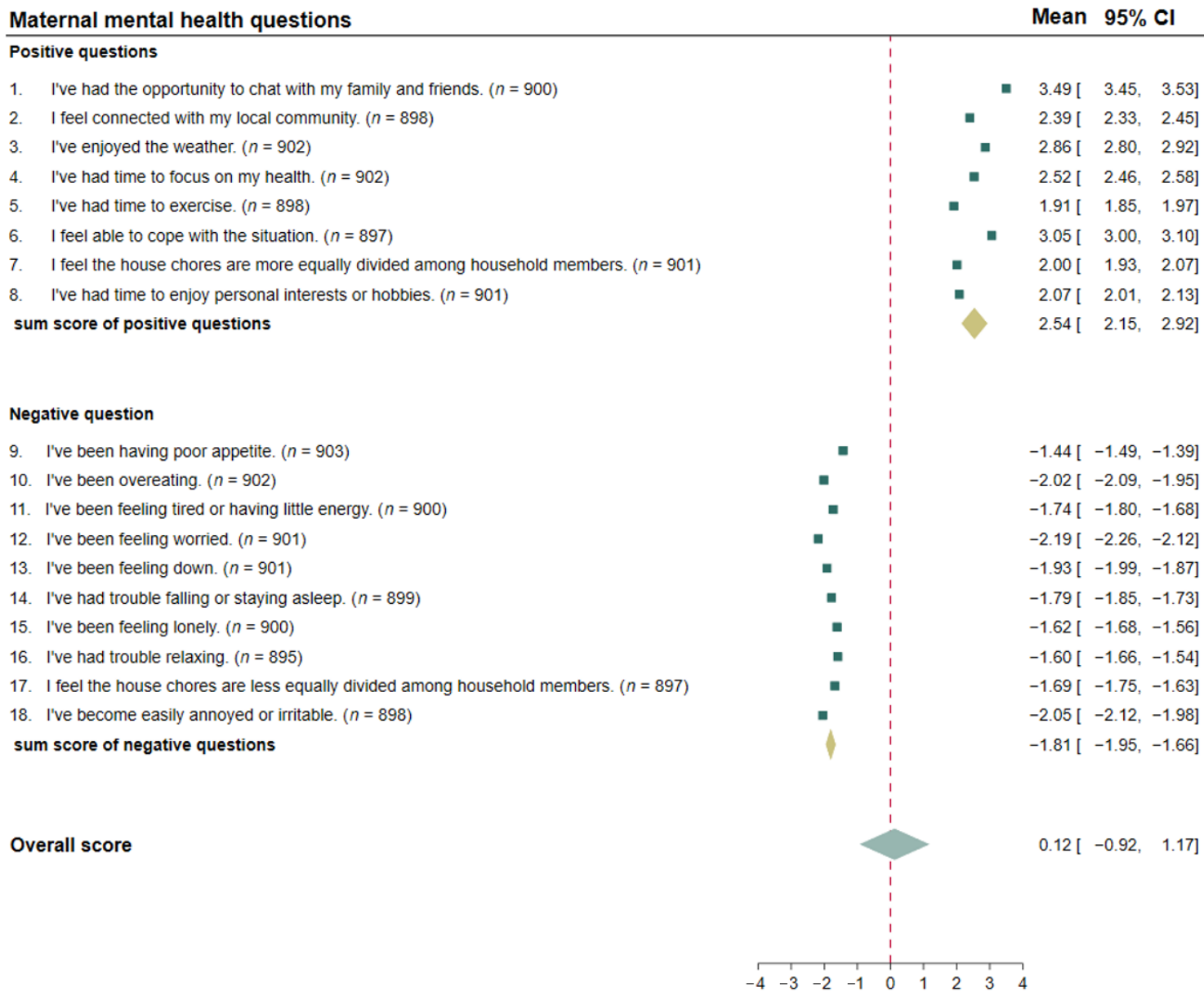

Figure 3. The estimated scores of the responses to the maternal mental health questions by forest plot.

\subsection{The Associated Factors of Maternal Mental Health during the COVID-19 Lockdown Period}

The results indicated mothers who had newborns aged less than six months, a household income per year of less than 16,130 USD, and practiced a relaxation technique had a significant positive effect on mood during the COVID-19 lockdown period, when other variables were controlled. On the other hand, the perceived impact of COVID-19 lockdown on "household ability to pay for rent/to make mortgage payments", "household ability to pay for other essentials, such as utilities and medication", "household crowding after lockdown", and "not going outside or doing outdoor activities" had a significant negative effect on maternal mental health during the COVID-19 lockdown period after being adjusted with the other variables (Table 4). The possible relationship between maternal mental health and influencing factors as a consequence of the COVID-19 lockdown is illustrated in Figure 4 . 


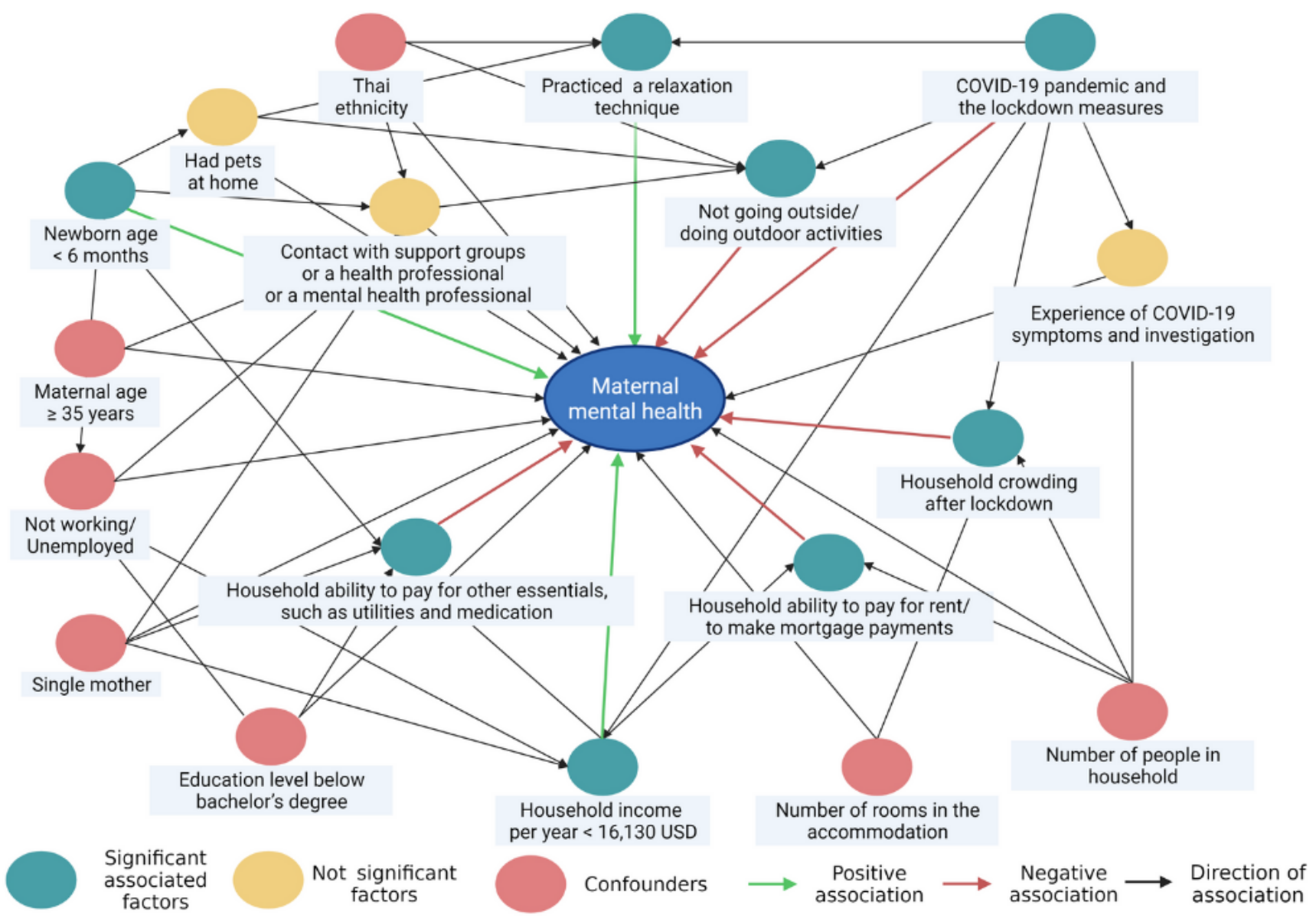

Figure 4. Relationship identification of maternal mental health during the COVID-19 nationwide lockdown period. Green ovals, yellow ovals, and pink ovals represent significant, not significant associated factors, and confounders, respectively. Green arrows indicate significant positive associations, whereas red arrows represent significant negative associations. Black arrows represent the direction of associations, which are not significant paths or confounding paths. The directed acyclic graphs were generated with publication licensed by BioRender, Toronto, Canada (Agreement number: QG237SORHP, 19 November 2021).

Table 4. Association between socio-demographics, perception of COVID-19 lockdown impacts, maternal activities, illness experience, and maternal mental health.

\begin{tabular}{|c|c|c|c|}
\hline Variables & $\beta$-coef. & $95 \%$ CI & $p$-Value \\
\hline Age $\geq 35$ years & -0.29 & -1.40 to 0.82 & 0.607 \\
\hline Newborn age $<6$ months & 1.14 & 0.13 to 2.15 & $0.026^{*}$ \\
\hline Thai ethnicity & -0.98 & -2.91 to 0.94 & 0.317 \\
\hline Single mother & 0.10 & -1.3 to 1.49 & 0.891 \\
\hline Education level below bachelor's degree & 0.85 & -0.47 to 2.17 & 0.205 \\
\hline Not working/Unemployed & -1.33 & -3.09 to 0.43 & 0.139 \\
\hline Household income per year $<16,130$ USD & 2.59 & 1.45 to 3.73 & $<0.001^{* *}$ \\
\hline Number of rooms in the accommodation & -0.10 & -0.27 to 0.08 & 0.272 \\
\hline Number of people in household & 0.38 & -0.06 to 0.82 & 0.091 \\
\hline Had pets at home & -0.40 & -1.42 to 0.62 & 0.439 \\
\hline \multicolumn{4}{|c|}{ Experience of COVID-19 symptoms and investigation } \\
\hline Had symptoms & 0.22 & -5.12 to 5.56 & 0.936 \\
\hline Any other member of household had symptoms & -0.05 & -6.4 to 6.3 & 0.988 \\
\hline Had been under investigated for COVID-19 & -0.42 & -2.24 to 1.4 & 0.653 \\
\hline
\end{tabular}


Table 4. Cont.

\begin{tabular}{|c|c|c|c|}
\hline Variables & $\beta$-coef. & $95 \%$ CI & $p$-Value \\
\hline \multicolumn{4}{|l|}{ COVID-19 lockdown impacts (moderate to major impacts) } \\
\hline Household's ability to pay for rent/to make mortgage payments & -1.59 & -2.81 to -0.36 & 0.011 * \\
\hline Household's ability to pay for food & -0.52 & -1.7 to 0.67 & 0.392 \\
\hline Household's ability to pay for other essentials, such as utilities and medication & -1.99 & -3.16 to -0.81 & $0.001 * *$ \\
\hline Impact on your employment/In what ways has your work been affected by COVID-19 & -0.32 & -1.37 to 0.74 & 0.554 \\
\hline Impact on your partner's employment/In what ways has their work been affected by COVID-19 & -0.54 & -1.57 to 0.49 & 0.305 \\
\hline Household crowding after lockdown & -3.46 & -4.86 to -2.06 & $<0.001 * *$ \\
\hline Not going outside/doing outdoor activities & -2.22 & -3.35 to -1.08 & $<0.001 * *$ \\
\hline \multicolumn{4}{|l|}{ Activity during COVID-19 lockdown (times/week) } \\
\hline Went shopping to the grocery store or pharmacy & -0.16 & -0.75 to 0.43 & 0.591 \\
\hline Went outside for a walk or for exercise & -0.06 & -0.54 to 0.42 & 0.796 \\
\hline Participated in an online activity & -0.21 & -0.6 to 0.18 & 0.291 \\
\hline Contact with a mother and baby support group or breastfeeding support group? & 0.01 & -0.98 to 1 & 0.980 \\
\hline Contact with a health professional (GP, Health Visitor, Midwife), in person, by phone or online & -0.21 & -0.74 to 0.32 & 0.443 \\
\hline Attended an online, phone, or in person appointment with a mental health professional & -1.48 & -3.96 to 1 & 0.241 \\
\hline Practiced a relaxation technique & 1.05 & 0.57 to 1.52 & $<0.001^{* *}$ \\
\hline Constant & 5.33 & -10.98 to 21.63 & 0.521 \\
\hline
\end{tabular}

$\beta$-coef. = Beta co-efficient; GP = General practitioner; 1.00 USD = 32.00 THB; Analyzed by multiple linear regression; ${ }^{*}$ Significant association at $p<0.05,{ }^{* *}$ Significant association at $p<0.001$.

\section{Discussion}

Maternal mental health problems are considered a major public health challenge and are recognized globally to have potential long-term consequences for mothers and infants [22]. During the first 12 months after childbirth, approximately $20 \%$ of mothers in developing countries and 13\% in Southeast Asian countries have clinical depression [23]. Additionally, postpartum women frequently experience other mental health problems, such as anxiety, insomnia, and psychological distress [24,25]. Recent cohort and meta-analysis studies showed that the prevalence of maternal depression and anxiety increased during the COVID-19 pandemic compared with previous estimates [26,27]. As reported, the nationwide lockdown, resulting in a disruption of healthcare services and social interaction, has aggravated the mental health problems of new mothers. Our study demonstrated that new mothers reported their feelings as 'high to some extent' most frequently for worrying $(44.9 \%)$, increased appetite $(40.4 \%)$, becoming easily annoyed or irritable $(39.1 \%)$, and feeling down (33.5\%), which were higher than those reported in studies before the pandemic $[24,25,28]$. Given the fact that the majority of new mothers $(82.7 \%)$ felt able to cope with their first lockdown situation, increasing awareness and support for maternal mental health should be advocated for to prevent this problem under the stress of repetitive lockdowns and outbreaks. This study aimed to explore the relationship between possible influencing factors and maternal mental health during the COVID-19 lockdown. According to the results, a quarter to half of the participant households had experienced significant impacts from the nationwide lockdown measures. Perceived moderate to major impacts on household overcrowding and households' ability to make payments of household expenses showed an independent negative effect on maternal mental health. Furthermore, not going outside or doing outdoor activities was significantly related to poor mental health. Being in a low-income household, having an infant under six months, and more frequent practice of a relaxation technique were independent positive predictors of maternal mental health.

The possible explanation for a positive effect of having an infant under six months might be related to the timing of maternity return to work in Thailand according to the Labour Protection Act and Thailand culture [29]. The return-to-work study in Thailand showed that new mothers intended to return to work within 6 months due to the 98-day maternity leave policy with full pay for employees covered by the Social Security Scheme, 
economic concerns, and fear of losing their job. Some of the traditional Thai postpartum practices, including food restrictions, activity restrictions, and staying at home, may also influence their decision to return to work since these practices are still common in modern Thai culture [30]. Furthermore, mothers working in the informal private sector were not covered by maternity leave policies and those in lower-level positions were particularly vulnerable [31]. Mothers returning to work face several obstacles, from the responsibilities of both caring for their children and work stresses, particularly in the context of an economic downturn caused by the COVID-19 pandemic. It was surprising that low-income was independently associated with positive maternal mental health, not only in our study, but also the study in the US that reported living in lower socioeconomic status expressed improved postpartum mood over the lockdown period [32]. Moreover, having a low income is a risk factor for maternal mental health as reported in the previous studies during the lockdown period $[18,20,21]$. The explanation of this finding in our study is uncertain. It is possible that the pandemic and the first lockdown measures had a limited effect on low-income households or a greater effect on middle- to high-income households in our setting.

Consistent with other studies, difficulties in paying general household expenses were significantly associated with negative maternal mental health in the lockdown situations. Nevertheless, trouble with the ability to afford food during the lockdown, which negatively affected mental health in UK new mothers [20], was not a significant factor in our population and the Chinese population [21]. These disparities may be explained by differences in social contexts between Asian countries and the United Kingdom, as well as by governmentsupportive measures. The perception of household crowding after the lockdown had a substantial negative effect on maternal mental health independently and did not directly depend on the number of rooms or members in the household. This result was also observed in previous studies either before or during the lockdown. This emphasizes that the role of partners and family in helping new mothers is not only in providing a personal space within the household, but also in the emotional support offered. Our study also highlighted that practicing relaxation techniques was a significant positive factor in maternal mental health during the lockdown, whereas a negative association was found between not going outside or doing outdoor activities and maternal mental health. The benefit of practicing relaxation techniques in reducing anxiety was also found in a recent study during the lockdown [33]. Furthermore, a recent review of clinical studies discovered that there was a preventive effect of practicing relaxation techniques on postpartum mood disturbances [34]. The potential benefits of relaxation techniques and enhanced outdoor activity on maternal mental health and promoting these activities in new mothers during the lockdown period should be advocated. Additional investigations are necessary to conclude the actual benefits regarding the type, frequency, and duration of activity for the particularistic population.

The COVID-19 pandemic and lockdown have disrupted healthcare and social interactions, resulting in a loss of health service contact, a lack of support from health experts, and loss of social participation. In this study, remote contacting support groups (e.g., a mother and baby support group) and healthcare professionals were investigated as potential activities used to cope with the lockdown impact. Nevertheless, there was no significant association between having contact with support groups or healthcare practitioners and maternal mental health in our study, whereas improved mental health was related to these activities in the China and UK studies. This different result might be explained by the population preferences and available healthcare services in the different countries. Since most mental health services are only available in secondary and tertiary healthcare settings in Thailand, remote contact with healthcare professionals via phone calls or online platforms for mental health issues is not a common practice and was not widely available during the first lockdown. Furthermore, our data were collected during the first lockdown in situations in which the COVID-19 was generally under control and the lockdown was temporary. As a result, these activities may have a limited impact on maternal mental health in our study. Further studies should be supported in view of the recent situation 
(following the third and fourth waves of the COVID-19 pandemic) to determine an effective response strategy and activity for promoting maternal mental health under the stress of repetitive lockdowns and increased economic pressures.

Many established studies have shown that mothers with newborns are more likely to suffer from higher stress, worries, anxiety, and depression during the postpartum period, particularly in the COVID-19 pandemic. Our study provided evidence of exacerbated mental health and determined its associated factors in Thai new mothers during the first lockdown period. Nonetheless, our study has several limitations that should be addressed. The survey data were collected promptly from a large group of mothers one month after the first lockdown ended and before the second wave of the COVID-19 pandemic in Thailand using both online and face-to-face interview surveys. It is possible that recall bias may exist because of the timing of the survey; however, using an online survey and face-to-face interviews may be an effective method for recruiting participants who represent a diversity of maternal socioeconomic backgrounds. Our study participants might not represent mothers with poor socioeconomic levels and lack of social support who are unable access to healthcare services and the internet (e.g., minority groups, hill-tribes, and migrants). Moreover, the limitation of the cross-sectional design should be considered when interpreting the reported association between maternal mental health and the associated factors. Because these variables were examined concurrently, the temporal relationship between them cannot be determined. Another limitation is that rates of worry or anxiety in new mothers during lockdown could not be compared to pre-pandemic rates since mental health problems were not routinely evaluated in our settings.

\section{Conclusions}

In conclusion, our survey of Thai new mothers indicated that mental health problems increased during the first COVID-19 pandemic lockdown compared to the pre-pandemic prevalence. Practiced relaxation techniques were found to have a beneficial effect on maternal mental health. Encouraging new mothers to go outdoors or participate in outdoor activities during the pandemic should be weighed against the risk of infection and the positive effects on their mental health. The negative effects of household overcrowding and difficulties in paying for general household expenses as a result of the lockdown necessarily require additional support, particularly under the stress of repetitive lockdowns and increased economic pressures.

Supplementary Materials: The following supporting information can be downloaded at: https:/ /www. mdpi.com/article/10.3390/ijerph19010347/s1, Table S1: Maternal mental health questionnaire reliability assessment, Table S2: Maternal mental health during the nationwide COVID-19 lockdown period.

Author Contributions: Conceptualization, W.S., K.O., P.S. and C.P.; data curation, W.S., K.O. and P.S.; formal analysis, W.S. and P.S.; funding acquisition, C.P.; investigation, W.S., C.P., K.O. and P.S.; methodology, W.S., K.O., C.P. and P.S.; project administration, W.S. and C.P.; resources, C.P.; software, W.S.; supervision, K.O. and P.S.; validation W.S., K.O. and P.S.; Visualization, W.S., K.O. and P.S.; writing-original draft, W.S.; writing - review and editing, K.O. and P.S. All authors have read and agreed to the published version of the manuscript.

Funding: The APC was funded by the Faculty of Medicine, Chiang Mai University, Thailand.

Institutional Review Board Statement: The study was conducted according to the guidelines of the Declaration of Helsinki, and approved by the Research Ethics Committee, Faculty of Medicine, Chiang Mai University (Study Code: COM-2563-07416).

Informed Consent Statement: Electronic and written informed consent was obtained from all subjects involved in the study.

Data Availability Statement: The data presented in this study are available on request from the correspondent author. 
Acknowledgments: Special thanks to Jonathan Wells, Mary Fewtrell, and the Population, Policy, and Practice Research and Teaching Programme, University College London (UCL). We would like to thank the mothers who provided the information for the project. We are also grateful to Mike Griffiths for English proofreading this manuscript.

Conflicts of Interest: The authors declare no conflict of interest.

\section{References}

1. Sen-Crowe, B.; McKenney, M.; Elkbuli, A. Social distancing during the COVID-19 pandemic: Staying home save lives. Am. J. Emerg. Med. 2020, 38, 1519-1520. [CrossRef] [PubMed]

2. Teslya, A.; Pham, T.M.; Godijk, N.G.; Kretzschmar, M.E.; Bootsma, M.C.J.; Rozhnova, G. Impact of self-imposed prevention measures and short-term government-imposed social distancing on mitigating and delaying a COVID-19 epidemic: A modelling study. PLoS Med. 2020, 17, e1003166. [CrossRef] [PubMed]

3. Lesourd, B.M. Nutrition and immunity in the elderly: Modification of immune responses with nutritional treatments. Am. J. Clin. Nutr. 1997, 66, 478s-484s. [CrossRef] [PubMed]

4. Agarwal, E.; Miller, M.; Yaxley, A.; Isenring, E. Malnutrition in the elderly: A narrative review. Maturitas 2013, 76, 296-302. [CrossRef]

5. Czeisler, M.É.; Lane, R.I.; Petrosky, E.; Wiley, J.F.; Christensen, A.; Njai, R.; Weaver, M.D.; Robbins, R.; Facer-Childs, E.R.; Barger, L.K.; et al. Mental Health, Substance Use, and Suicidal Ideation During the COVID-19 Pandemic-United States, June 24-30, 2020. MMWR Morb. Mortal. Wkly. Rep. 2020, 69, 1049-1057. [CrossRef]

6. Wang, C.; Pan, R.; Wan, X.; Tan, Y.; Xu, L.; Ho, C.S.; Ho, R.C. Immediate Psychological Responses and Associated Factors during the Initial Stage of the 2019 Coronavirus Disease (COVID-19) Epidemic among the General Population in China. Int. J. Environ. Res. Public Health 2020, 17, 1729. [CrossRef]

7. Qiu, J.; Shen, B.; Zhao, M.; Wang, Z.; Xie, B.; Xu, Y. A nationwide survey of psychological distress among Chinese people in the COVID-19 epidemic: Implications and policy recommendations. Gen. Psychiatry 2020, 33, e100213. [CrossRef]

8. Alkhamees, A.A.; Alrashed, S.A.; Alzunaydi, A.A.; Almohimeed, A.S.; Aljohani, M.S. The psychological impact of COVID-19 pandemic on the general population of Saudi Arabia. Compr. Psychiatry 2020, 102, 152192. [CrossRef]

9. Adams-Prassl, A.; Boneva, T.; Golin, M.; Rauh, C. The Impact of the Coronavirus Lockdown on Mental Health: Evidence from the US; University of Cambridge: Cambridge, UK, 2020. [CrossRef]

10. Pierce, M.; Hope, H.; Ford, T.; Hatch, S.; Hotopf, M.; John, A.; Kontopantelis, E.; Webb, R.; Wessely, S.; McManus, S.; et al. Mental health before and during the COVID-19 pandemic: A longitudinal probability sample survey of the UK population. Lancet Psychiatry 2020, 7, 883-892. [CrossRef]

11. Biroli, P.; Bosworth, S.; Della Giusta, M.; Di Girolamo, A.; Jaworska, S.; Vollen, J. Family Life in Lockdown. Front. Psychol. 2021, 12, 687570. [CrossRef]

12. Giuntella, O.; Hyde, K.; Saccardo, S.; Sadoff, S. Lifestyle and mental health disruptions during COVID-19. Proc. Natl. Acad. Sci. USA 2021, 118, e2016632118. [CrossRef] [PubMed]

13. Piankusol, C.; Sirikul, W.; Ongprasert, K.; Siviroj, P. Factors Affecting Breastfeeding Practices under Lockdown during the COVID-19 Pandemic in Thailand: A Cross-Sectional Survey. Int. J. Environ. Res. Public Health 2021, 18, 8729. [CrossRef] [PubMed]

14. Banks, J.; Xu, X. The Mental Health Effects of the First Two Months of Lockdown and Social Distancing during the COVID-19 Pandemic in the UK; IFS Working Paper W20/16; Institute for Fiscal Studies (IFS): London, UK, 2020. [CrossRef]

15. Brooks, S.K.; Webster, R.K.; Smith, L.E.; Woodland, L.; Wessely, S.; Greenberg, N.; Rubin, G.J. The psychological impact of quarantine and how to reduce it: Rapid review of the evidence. Lancet 2020, 395, 912-920. [CrossRef]

16. Riley, V.; Ellis, N.; Mackay, L.; Taylor, J. The impact of COVID-19 restrictions on women's pregnancy and postpartum experience in England: A qualitative exploration. Midwifery 2021, 101, 103061. [CrossRef]

17. Collin, J.; Byström, E.; Carnahan, A.; Ahrne, M. Public Health Agency of Sweden's Brief Report: Pregnant and postpartum women with severe acute respiratory syndrome coronavirus 2 infection in intensive care in Sweden. Acta Obstet. Gynecol. Scand. 2020, 99, 819-822. [CrossRef]

18. Perzow, S.E.D.; Hennessey, E.-M.P.; Hoffman, M.C.; Grote, N.K.; Davis, E.P.; Hankin, B.L. Mental health of pregnant and postpartum women in response to the COVID-19 pandemic. J. Affect. Disord. Rep. 2021, 4, 100123. [CrossRef]

19. Vazquez-Vazquez, A.; Dib, S.; Rougeaux, E.; Wells, J.C.; Fewtrell, M.S. The impact of the COVID-19 lockdown on the experiences and feeding practices of new mothers in the UK: Preliminary data from the COVID-19 New Mum Study. Appetite 2021, 156, 104985. [CrossRef] [PubMed]

20. Dib, S.; Rougeaux, E.; Vázquez-Vázquez, A.; Wells, J.C.K.; Fewtrell, M. Maternal mental health and coping during the COVID-19 lockdown in the UK: Data from the COVID-19 New Mum Study. Int. J. Gynecol. Obstet. 2020, 151, 407-414. [CrossRef] [PubMed]

21. Wei, Z.; Gao, M.Y.; Fewtrell, M.; Wells, J.; Yu, J.Y. Maternal mental health and well-being during the COVID-19 pandemic in Beijing, China. World J. Pediatr. 2021, 17, 280-289. [CrossRef] [PubMed]

22. World Health Organization. Maternal Mental Health and Child Health and Development in Low and Middle Income Countries: Report of the Meeting, Geneva, Switzerland, 30 January-1 February, 2008; World Health Organization: Geneva, Switzerland, 2008.

23. Wang, Z.; Liu, J.; Shuai, H.; Cai, Z.; Fu, X.; Liu, Y.; Xiao, X.; Zhang, W.; Krabbendam, E.; Liu, S.; et al. Mapping global prevalence of depression among postpartum women. Transl. Psychiatry 2021, 11, 543. [CrossRef] [PubMed] 
24. Dennis, C.L.; Falah-Hassani, K.; Shiri, R. Prevalence of antenatal and postnatal anxiety: Systematic review and meta-analysis. Br. J. Psychiatry 2017, 210, 315-323. [CrossRef]

25. Sedov, I.D.; Cameron, E.E.; Madigan, S.; Tomfohr-Madsen, L.M. Sleep quality during pregnancy: A meta-analysis. Sleep Med. Rev. 2018, 38, 168-176. [CrossRef]

26. Racine, N.; Hetherington, E.; McArthur, B.A.; McDonald, S.; Edwards, S.; Tough, S.; Madigan, S. Maternal depressive and anxiety symptoms before and during the COVID-19 pandemic in Canada: A longitudinal analysis. Lancet Psychiatry 2021, 8, 405-415. [CrossRef]

27. Yan, H.; Ding, Y.; Guo, W. Mental Health of Pregnant and Postpartum Women During the Coronavirus Disease 2019 Pandemic: A Systematic Review and Meta-Analysis. Front. Psychol. 2020, 11, 3324. [CrossRef] [PubMed]

28. Woody, C.A.; Ferrari, A.J.; Siskind, D.J.; Whiteford, H.A.; Harris, M.G. A systematic review and meta-regression of the prevalence and incidence of perinatal depression. J. Affect. Disord. 2017, 219, 86-92. [CrossRef] [PubMed]

29. Topothai, C.; Tangcharoensathien, V. Achieving global targets on breastfeeding in Thailand: Gap analysis and solutions. Int. Breastfeed. J. 2021, 16, 38. [CrossRef] [PubMed]

30. Kaewsarn, P.; Moyle, W.; Creedy, D. Traditional postpartum practices among Thai women. J. Adv. Nurs. 2003, 41, 358-366. [CrossRef]

31. Aikawa, T.; Pavadhgul, P.; Chongsuwat, R.; Sawasdivorn, S.; Boonshuyar, C. Maternal return to paid work and breastfeeding practices in Bangkok, Thailand. Asia Pac. J. Public Health 2015, 27, Np1253-Np1262. [CrossRef] [PubMed]

32. Silverman, M.E.; Burgos, L.; Rodriguez, Z.I.; Afzal, O.; Kalishman, A.; Callipari, F.; Pena, Y.; Gabay, R.; Loudon, H. Postpartum mood among universally screened high and low socioeconomic status patients during COVID-19 social restrictions in New York City. Sci. Rep. 2020, 10, 22380. [CrossRef]

33. Fullana, M.A.; Hidalgo-Mazzei, D.; Vieta, E.; Radua, J. Coping behaviors associated with decreased anxiety and depressive symptoms during the COVID-19 pandemic and lockdown. J. Affect. Disord. 2020, 275, 80-81. [CrossRef]

34. Oyarzabal, E.A.; Seuferling, B.; Babbar, S.; Lawton-O’Boyle, S.; Babbar, S. Mind-Body Techniques in Pregnancy and Postpartum. Clin. Obstet. Gynecol. 2021, 64, 683-703. [CrossRef] [PubMed] 